\title{
Magnetic excitations in coupled Haldane spin chains near the quantum critical point
}

\author{
A. Zheludev \\ Department of Physics, Brookhaven National Laboratory, Upton, New York 11973-5000 \\ T. Masuda, ${ }^{*}$ I. Tsukada, ${ }^{\dagger}$ Y. Uchiyama ${ }^{\dagger}$ and K. Uchinokura* \\ Department of Applied Physics, The University of Tokyo, 6th Engineering Building, 7-3-1 Bunkyo-ku, Tokyo 113-8656, Japan \\ P. Böni \\ Paul Scherrer Institut, 5232 Villigen PSI, Switzerland
}

S.-H. Lee

NIST Center for Neutron Research, National Institute of Standards and Technology, Gaithersburg, Maryland 20899

(Received 26 January 2000; revised manuscript received 2 May 2000)

\begin{abstract}
Two quasi-one-dimensional $S=1$ quantum antiferromagnetic materials, $\mathrm{PbNi}_{2} \mathrm{~V}_{2} \mathrm{O}_{8}$ and $\mathrm{SrNi}_{2} \mathrm{~V}_{2} \mathrm{O}_{8}$, are studied by inelastic neutron scattering on powder samples. A simple model Hamiltonian is proposed for the two systems and used to analyze the data. Quantitative information on in-chain and interchain interactions obtained from this analysis is discussed in the context of qualitatively different ground-state properties: magnetic long-range order in $\mathrm{SrNi}_{2} \mathrm{~V}_{2} \mathrm{O}_{8}$ and disordered "spin liquid" Haldane-gap state in $\mathrm{PbNi}_{2} \mathrm{~V}_{2} \mathrm{O}_{8}$.
\end{abstract}

\section{INTRODUCTION}

After two decades of intensive theoretical and experimental studies, one-dimensional (1D) Heisenberg quantum antiferromagnets $(\mathrm{AF})$ are now rather well understood. A great deal of work has been done on integer-spin systems that have a spin-liquid ground state and a famous Haldane gap in the magnetic excitation spectrum. ${ }^{1,2}$ The focus in quantum magnetism has now shifted towards studies of more complex phenomena, that include interchain interactions, spin-lattice coupling, and/or spin-vacancies and substitutions. Of particular current interest is the quantum phase transition between spin-liquid (non-magnetic) and ordered states. This type of transition in gapped 1D systems occurs as 3D magnetic interactions and/or magnetic anisotropy are increased beyond certain threshold values. Their effect is to lower the energy of excitations at certain points in reciprocal space, and ultimately induce a soft-mode transition to a Néel-like ordered structure. An example of such behavior is found in the extensively studied $\mathrm{CsNiCl}_{3}$ compound. ${ }^{3-6}$ The corresponding phase diagram has been worked out by several authors, including Sakai and Takahashi ${ }^{7}$ (Fig. 1).

The most direct way to observe such a transition experimentally is by looking at a series of isostructural compounds with slightly different interchain coupling constants or anisotropy terms. The problem is that most known quasi-1D $S=1$ AF materials are otherwise deep inside the spin-liquid area of the phase diagram (good 1D systems), or obviously in the 3D Néel-like or $X Y$-like ordered phases (Fig. 1). $\mathrm{CsNiCl}_{3}$ and related compounds ${ }^{3,5,8}$ are perhaps the only systems close to the phase boundary that have been extensively studied to date. Unfortunately, these compounds order in three dimensions at low temperature, and have no isostructural counterpart with a spin-liquid ground state. Only about a year ago the first quasi-1-D integer-spin AF that is still in the spin-liquid state, but is on the verge of a $3 \mathrm{D}$ ordering instability, was characterized. ${ }^{9}$ This Haldane-gap material, $\mathrm{PbNi}_{2} \mathrm{~V}_{2} \mathrm{O}_{8}$ is so close to the phase boundary that LRO, absent in the pure compound, can be induced by spinvacancy substitution. ${ }^{9}$ Moreover, an isostructural undoped system, namely $\mathrm{SrNi}_{2} \mathrm{~V}_{2} \mathrm{O}_{8}$ does order in three dimensions at low temperatures. This opens a unique opportunity for comparative studies of two very similar quasi-1D materials with vastly different ground-state properties. Regrettably, sufficiently large single crystals of either material are not available to date, and for now one has to content with measurements on powder samples.

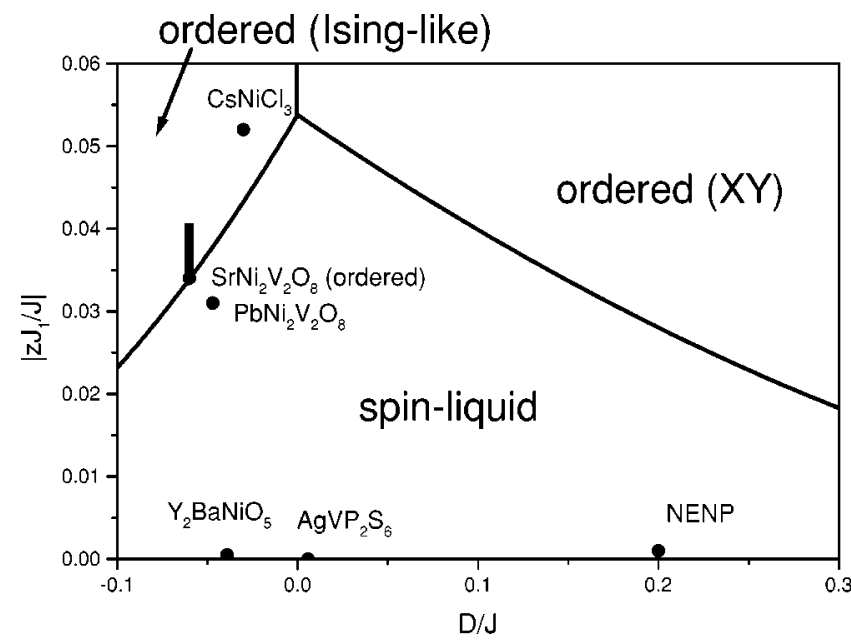

FIG. 1. $D-J_{1}$ Phase diagram for weakly coupled Haldane spin chains (Ref. 7), showing the location of some well-characterized quasi-1D $S=1$ systems. For each material $J_{1}$ is scaled by the coordination number. $\mathrm{CsNiCl}_{3}$ data from Ref. 4; $\mathrm{Y}_{2} \mathrm{BaNiO}_{5}$ data from Ref. 27; NENP data from Ref. 28; $\mathrm{AgVP}_{2} \mathrm{~S}_{6}$ data from Ref. 29. $\mathrm{PbNi}_{2} \mathrm{~V}_{2} \mathrm{O}_{8}$ and $\mathrm{SrNi}_{2} \mathrm{~V}_{2} \mathrm{O}_{8}$ are placed based on the results of this work. The vertical bar indicates that the $\mathrm{SrNi}_{2} \mathrm{~V}_{2} \mathrm{O}_{8}$ compound must be actually deeper in the ordered phase than suggested by our analysis. 
(a)
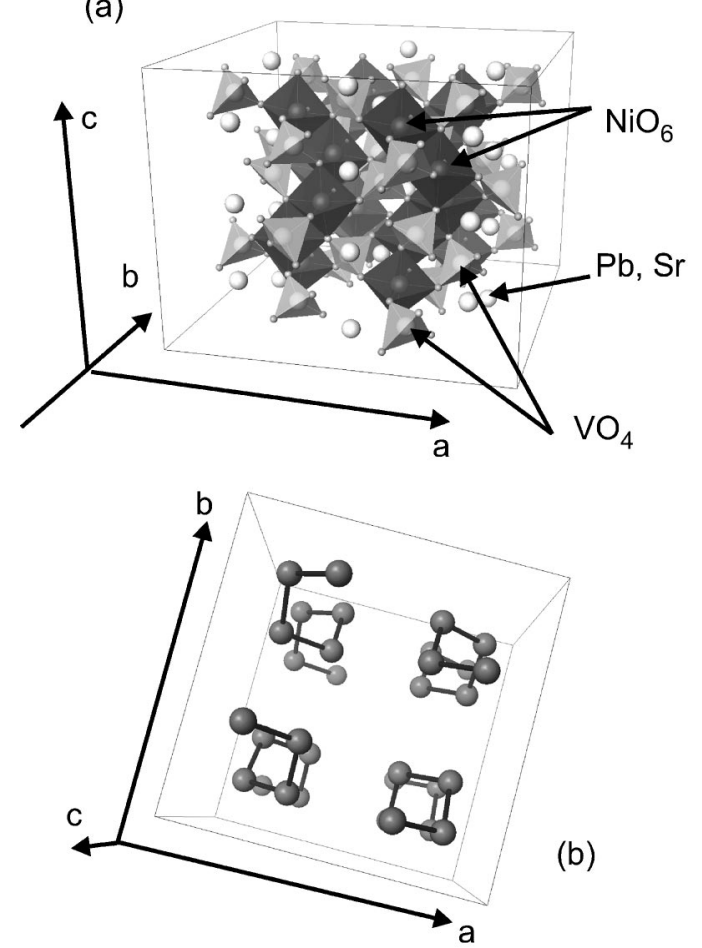

FIG. 2. (a) Crystal structure on $\mathrm{PbNi}_{2} \mathrm{~V}_{2} \mathrm{O}_{8}$ and $\mathrm{SrNi}_{2} \mathrm{~V}_{2} \mathrm{O}_{8}$. The magnetic chains are formed by edge-sharing $\mathrm{NiO}_{6}$ octahedra (dark gray) and are bridged by $\mathrm{VO}_{4}$ tetrahedra (light gray). (b) A perspective view of the spiral-shaped $\mathrm{Ni}^{2+}$-chains in $\mathrm{PbNi}_{2} \mathrm{~V}_{2} \mathrm{O}_{8}$ and $\mathrm{SrNi}_{2} \mathrm{~V}_{2} \mathrm{O}_{8}$.

The magnetic properties of both $\mathrm{PbNi}_{2} \mathrm{~V}_{2} \mathrm{O}_{8}$ and $\mathrm{SrNi}_{2} \mathrm{~V}_{2} \mathrm{O}_{8}$ are due to spin $S=1$ octahedrally coordinated $\mathrm{Ni}^{2+}$ ions, while the $\mathrm{V}^{5+}$ sites are presumed to be nonmagnetic. The crystal structure, visualized in Fig. 2(a), is tetragonal, space group $I 41 \mathrm{~cd}$, with lattice constants $a=12.249(3) \AA, \quad c=8.354(2) \AA$ for $\mathrm{PbNi}_{2} \mathrm{~V}_{2} \mathrm{O}_{8},{ }^{10}$ and $a=12.1617 \AA, c=8.1617 \AA$ for $\mathrm{SrNi}_{2} \mathrm{~V}_{2} \mathrm{O}_{8},{ }^{11}$ respectively. The magnetic Ni sites are arranged in peculiar spiral-shaped chains that run along the unique crystal axis, as shown in Fig. 2(b). Even though all nearest-neighbor Ni-Ni bonds are crystallographically equivalent, the spin spirals have a step-4 periodicity. The dominant magnetic interaction is antiferromagnetic, between nearest-neighbor $\mathrm{Ni}^{2+}$ spins within each chain. The corresponding exchange constant, $J \approx 8.2 \mathrm{meV}$ in both systems, was deduced from the high-temperature part of the experimental $\chi(T)$ curves. $^{9}$ The ground state of $\mathrm{PbNi}_{2} \mathrm{~V}_{2} \mathrm{O}_{8}$ is a Haldane singlet, and the excitation spectrum has an energy gap, as unambiguously shown in lowtemperature $\chi(T)$ and $C(T)$ measurements. The energy gaps, 1.2 and $2.2 \mathrm{meV}$, for excitations polarized along, and perpendicular to the chain axis, respectively, were accurately determined in high-field magnetization studies. The observed anisotropy of the spin gap is attributed to single-ion easyaxis magnetic anisotropy on the $\mathrm{Ni}$ sites $D \approx-0.23 \mathrm{meV}$.

Unlike $\mathrm{PbNi}_{2} \mathrm{~V}_{2} \mathrm{O}_{8}, \mathrm{SrNi}_{2} \mathrm{~V}_{2} \mathrm{O}_{8}$ orders magnetically in three dimensions at $T_{\mathrm{N}}=7 \mathrm{~K}$. The magnetic structure has not been determined to date, but, according to bulk measurements, is of a weak-ferromagnetic type, with a dominant antiferromagnetic component. The ordered staggered moment is along the unique $c$ crystallographic axis. The weak-ferro- magnet distortion of this Néel (collinear) spin arrangement is attributed to the presence of weak Dzyaloshinskii-Moriya off-diagonal exchange interactions in the noncentric crystal.

Preliminary inelastic neutron-scattering studies ${ }^{9}$ provided an estimate for the interchain interaction strength. Only a limited amount of neutron data are available for $\mathrm{PbNi}_{2} \mathrm{~V}_{2} \mathrm{O}_{8}$, and, to date, none for $\mathrm{SrNi}_{2} \mathrm{~V}_{2} \mathrm{O}_{8}$. The present paper deals with more extensive comparative inelastic neutron-scattering studies of both materials.

\section{A MODEL FOR MAGNETIC INTERACTIONS}

The main problem with interpreting inelastic neutronscattering data from powder samples is an effective loss of information upon spherical averaging. Indeed, the quantity of interest is the dynamic structure factor $S(Q, \omega)$ that for each channel of spin polarization is a scalar function in fourdimensional $E-\boldsymbol{Q}$ space. In a powder sample one measures the spherical average of this function, a scalar function defined in two-dimensional space:

$$
\begin{aligned}
S_{\text {powder }}(Q, \omega)= & \frac{1}{4 \pi} \int d \phi \sin \theta d \theta S([Q \sin \theta \cos \phi, \\
& Q \sin \theta \sin \phi, Q \cos \theta], \omega) .
\end{aligned}
$$

The transformation $S(\boldsymbol{Q}, \omega) \rightarrow S_{\text {powder }}(Q, \omega)$ is not reversible. The only way the full correlation function can be extracted from the experiment is by assuming some parametrized model and fitting it to the measured $S_{\text {powder }}(Q, \omega)$. Prior to reporting our experimental findings, we shall briefly discuss the magnetic interactions that may play an important role in the physics of $\mathrm{PbNi}_{2} \mathrm{~V}_{2} \mathrm{O}_{8}$ and $\mathrm{SrNi}_{2} \mathrm{~V}_{2} \mathrm{O}_{8}$, and construct a model spin Hamiltonian for these systems.

As mentioned in the introduction, all previous studies point to that the dominant magnetic interaction is the antiferromagnetic coupling $J$ between nearest-neighbor spins $(2.78 \AA)$ within each spiral-shaped chain. It is also clear that taking the expectedly weaker interchain interactions into account is crucial to understanding the static and dynamic properties. In our previous work (Ref. 9) we assumed that the dominant interchain coupling is between pairs of $\mathrm{Ni}^{2+}$ ions from adjacent chains, such that both interacting spins have the same $c$-axis fractional cell coordinate, and that each $\mathrm{Ni}$ site is coupled to four other sites. The resulting coupling topology is schematized in Fig. 3(a). Below we make use of a different model, visualized in Fig. 3(b). Here one assumes interactions along the shortest interchain $\mathrm{Ni}-\mathrm{Ni}$ bond $(5.0 \AA)$. Coupled $\mathrm{Ni}^{2+}$ ions are offset relative to each other by $c / 4$ along the chain axis. We shall denote the corresponding exchange constant as $J_{1}$. It is clear that in a powder experiment on a quasi-1-D system, one cannot hope to unambiguously determine the actual interchain coupling routes, as for low-energy excitations only the effective mean-field random-phase approximation (RPA) chain-coupling constant will be relevant. The two models will result in virtually identical dispersion relations, and the only difference will be in how one assigns microscopic exchange constants based on the measured transverse bandwidth of magnetic excitations. While it makes no real difference which model is actually used, we chose to switch to the second model, as it appears to be somewhat better justified from the structural point of 
(A)

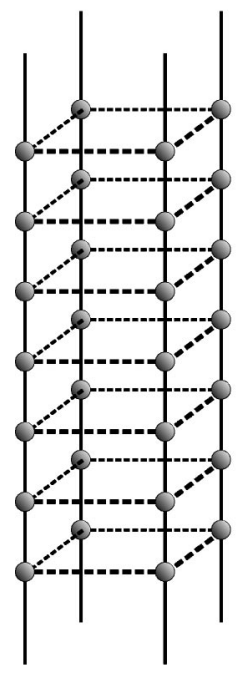

(B)

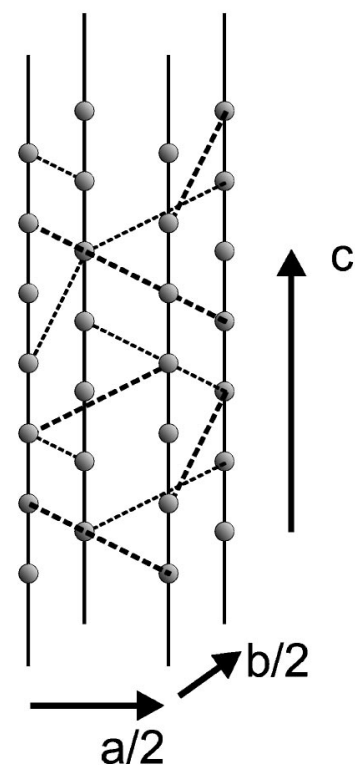

FIG. 3. Topology of magnetic interactions $\mathrm{PbNi}_{2} \mathrm{~V}_{2} \mathrm{O}_{8}$ and $\mathrm{SrNi}_{2} \mathrm{~V}_{2} \mathrm{O}_{8}$. Interchain bonds are shown as dashed lines. Solid lines are in-chain Ni-Ni bonds. (a): Model used in Ref. 9. (b): Model used in the present paper.

view. Indeed, it assumes coupling between nearest-neighbor (as opposed to next-nearest neighbor) Ni sites from adjacent chains. In addition, unlike in the previous model, where the actual exchange route is not clear, the coupled spins in the new model are bridged by $\mathrm{VO}_{4}$ tetrahedra, suggesting a possible superexchange pathway.

Magnetic anisotropy is clearly manifest in bulk susceptibility and magnetization measurements, and must be explicitly included in the spin Hamiltonian. Because of the strong dispersion along the chain axis, all the low-energy response of each chain is concentrated at wave vectors close to the 1-D AF zone center. In this long-wavelength limit, two-ion anisotropy of in-chain interactions and single-ion anisotropy associated with individual spins cannot be distinguished. In our model we shall therefore combine the two effects into an effective single-ion term:

$$
\hat{H}_{\text {single-ion }}=D \sum_{i, k}\left(S_{i, k}^{z}\right)^{2} .
$$

Here $D$ is the anisotropy constant $(D<0=$ easy-axis $)$ and $S_{i, k}$ is the spin operator for site $i$ in chain $k$. The choice of the anisotropy axis along the chain direction is based on previous bulk magnetic studies. Two-ion anisotropy being effectively included in the single-ion term, in-chain exchange interactions will be assumed to be isotropic:

$$
\hat{H}_{\text {in-chain }}=J \sum_{i, k} S_{i, k} S_{i+1, k} .
$$

The magnitude of dispersion perpendicular to the chain axis is expected to be rather small, so the entire range of wavevector transfers in the $(a, b)$ plane will influence the lowenergy properties. For this reason, two-ion anisotropy of $J_{1}$, unlike that of $J$, is a relevant parameter and should be considered. The interchain coupling term in the Hamiltonian will have the form:

$$
\begin{aligned}
\hat{H}_{\text {inter-chain }}= & \sum_{i, i^{\prime}, k, k^{\prime}}\left[J_{1, \|} S_{i, k}^{z} S_{i^{\prime}, k^{\prime}}^{z}\right. \\
& \left.+J_{1, \perp}\left\{S_{i, k^{\prime}}^{x} S_{i^{\prime}, k^{\prime}}^{x}+S_{i, k^{\prime}}^{y} S_{i^{\prime}, k^{\prime}}^{y}\right\}\right] .
\end{aligned}
$$

Here the sum is taken over pairs of nearest-neighbor spins from adjacent chains.

Once again, we stress that one cannot hope to determine all the details of magnetic interactions in a system from a powder experiment. Any data analysis will not be a unique interpretation, and will depend on the model used. As will be discussed below, however, the simple Hamiltonian proposed above can reproduce the previously published bulk data, as well as the new inelastic neutron-scattering results with a minimal number of adjustable parameters.

\section{EXPERIMENTAL}

Inelastic neutron-scattering studies of $\mathrm{PbNi}_{2} \mathrm{~V}_{2} \mathrm{O}_{8}$ and $\mathrm{SrNi}_{2} \mathrm{~V}_{2} \mathrm{O}_{8}$ powder samples (about $10 \mathrm{~g}$ each) were carried out in two series of experiments. The conventional three-axis technique was used to measure certain characteristic constant- $E$ and constant- $Q$ scans. These measurements were performed at the TASP and Druchal spectrometers at the continuous spallation source SINQ at Paul Scherrer Institute. Neutrons of a fixed final energy of $5 \mathrm{meV}$ or $8 \mathrm{meV}$ were used with pyrolytic graphite monochromator and analyzer, and a typical (open) $-80^{\prime}-80^{\prime}-(\text { open })^{\prime}$ collimation setup. For some scans a horizontally focusing analyzer was used to increase the useful scattered intensity, in which case no collimators were used after the sample. In most of the measurements a flat analyzer was employed instead. In the $5 \mathrm{meV}$ final configuration a Be filter was inserted after the sample to suppress higher-order beam contamination. The spectrum of neutrons emerging from the guide is such that no higherorder filter was required for the $8 \mathrm{meV}$-final measurements.

In the second series of experiments we took advantage of the area-sensitive detector (ASD) three-axis setup available for the NG-5 "SPINS" spectrometer installed at the National Institute of Standards and Technology. In special cases when a large domain of $E-Q$ space needs to be surveyed in a spherically symmetric sample (such as powder) this technique can provide an almost tenfold increase of data collection rate, as compared to the standard three-axis geometry, without any penalty in resolution. In our experiments on $\mathrm{PbNi}_{2} \mathrm{~V}_{2} \mathrm{O}_{8}$ and $\mathrm{SrNi}_{2} \mathrm{~V}_{2} \mathrm{O}_{8}$ we utilized a PG monochromator and a (open) $-80^{\prime}-80^{\prime}-80^{\prime}$ (radial) array of collimators. The measurements were done in the fixed-finalenergy mode, with the central blade of the composite analyzer tuned to $E_{\mathrm{f}}=3.125 \mathrm{meV}$. In the experiment scattering events with final neutron energies in the range $E_{\mathrm{f}}$ $\pm 0.4 \mathrm{meV}$ are registered simultaneously. To suppress higher-order beam contamination we used a Be-O filter after the sample. The data were taken for momentum and energy transfers of up to $2.5 \AA^{-1}$ and $7 \mathrm{meV}$, respectively.

In both series of experiments the sample environment was a standard "ILL-orange" cryostat, and the temperature range $1.5-30 \mathrm{~K}$ was covered. The background was measured by 


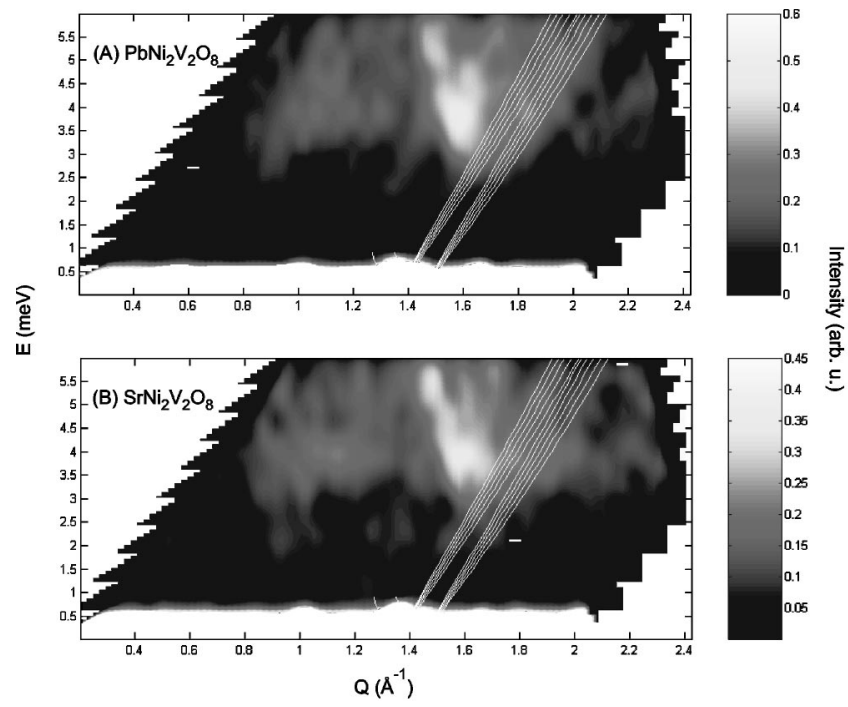

FIG. 4. False-color plot of inelastic neutron-scattering intensities measured in $\mathrm{PbNi}_{2} \mathrm{~V}_{2} \mathrm{O}_{8}$ (a) and $\mathrm{SrNi}_{2} \mathrm{~V}_{2} \mathrm{O}_{8}$ (b) at $T=1.5 \mathrm{~K}$ using an area-sensitive detector setup. The background has been subtracted, as described in the text. Shaded areas indicate the location of possible spurions.

repeating some scans with the analyzer moved away from its elastic position by $10^{\circ}$. In the SPINS experiment the background due to air scattering was separately measured with the sample removed from the spectrometer.

\section{EXPERIMENTAL RESULTS}

\section{A. Results obtained at low temperatures}

The inelastic neutron-scattering intensities measured in $\mathrm{PbNi}_{2} \mathrm{~V}_{2} \mathrm{O}_{8}$ and $\mathrm{SrNi}_{2} \mathrm{~V}_{2} \mathrm{O}_{8}$ at $T=1.2 \mathrm{~K}$ with the ASD setup

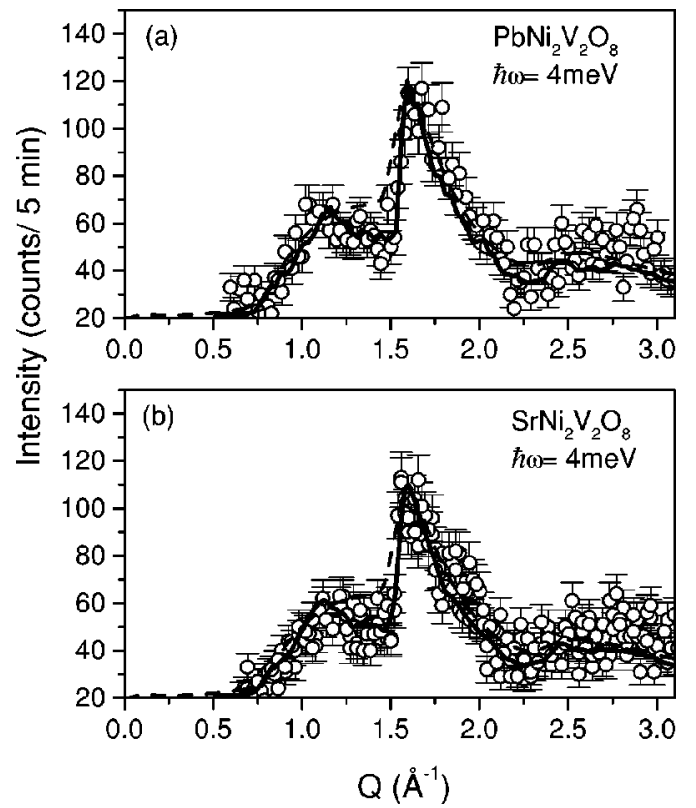

FIG. 5. Constant-energy scans measured in $\mathrm{PbNi}_{2} \mathrm{~V}_{2} \mathrm{O}_{8}$ (a) and $\mathrm{SrNi}_{2} \mathrm{~V}_{2} \mathrm{O}_{8}$ (b) powder samples at $T=2 \mathrm{~K}$ in the standard three-axis mode. The solid lines are simulations based on parameters obtained in a global fit to the ASD data, as described in the text. Dashed lines are similar simulations for noninteracting spiral-shaped spin chains.
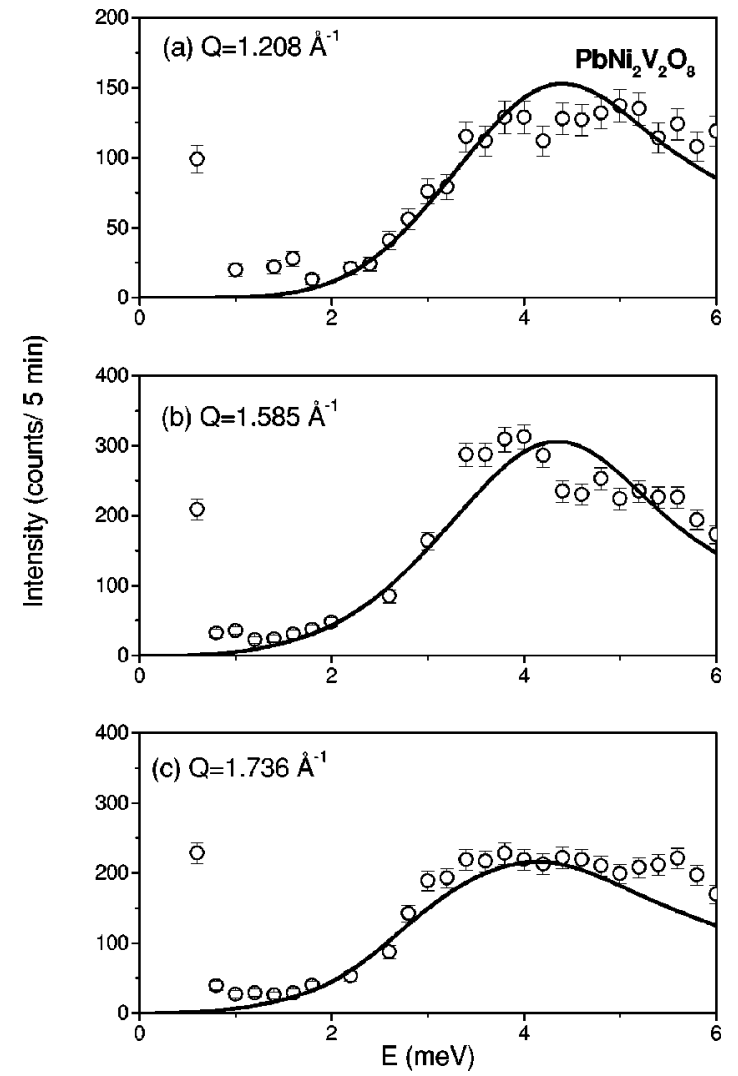

FIG. 6. Typical constant- $Q$ scans measured in $\mathrm{PbNi}_{2} \mathrm{~V}_{2} \mathrm{O}_{8}$ powder sample at $T=2 \mathrm{~K}$ in the standard three-axis mode. The solid lines are as in Fig. 5.

are shown in the false-color plot in Fig. 4 (background subtracted). The resolution of the area-sensitive detector is greater than the actual energy and wave vector resolution of the spectrometer $\left(0.11 \mathrm{meV}\right.$ and $0.023 \AA^{-1}$ at $\left.\hbar \omega=0\right)$. The customary procedure is to resample the mesh data to a coarser grid. Instead, for visualization purposes, the data in Fig. 4 were smeared using a fixed-resolution $0.5 \mathrm{meV}$ $\times 0.06 \AA^{-1}$ full width at half maximum Gaussian filter. As reported previously, ${ }^{9}$ constant-energy scans measured in $\mathrm{PbNi}_{2} \mathrm{~V}_{2} \mathrm{O}_{8}$ at $4 \mathrm{meV}$ energy transfer have a peculiar and very characteristic shape. Such scans were collected at $T$ $=2 \mathrm{~K}$ for both materials, using the $8 \mathrm{meV}$-final/flat analyzer setup SINQ. These scans are shown in Fig. 5. In addition, representative constant- $Q$ scans were also measured in this configuration, and are shown in Figs. 6 and 7.

Despite the different ground states, the main features of the powder-averaged dynamic cross section for the two materials are quite similar. This, however, is not particularly surprising. The dynamic structure factor $S(Q, \omega)$ is severely smeared out by the spherical averaging that occurs in a powder sample. The main features are defined by the form factor of each spiral-shaped chain, and by the steep dispersion along the chain axis, expected to be almost identical in the two systems. The significant difference between the two materials is expected to be in the magnitude of the interchain interactions, and possibly single-ion anisotropy. These effects are much more difficult to observe, as they only influence the weak spin-wave dispersion in the $(a, b)$ crystallographic plane. Our data do in fact contain relevant information on the transverse dispersion of spin excitations, 

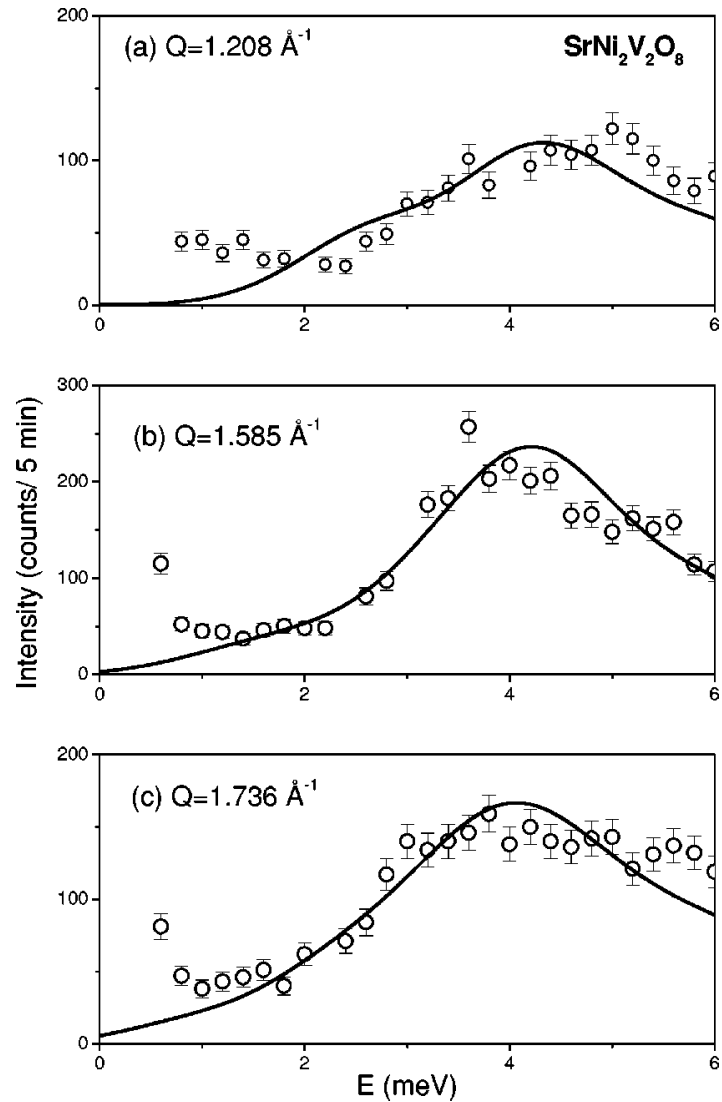

FIG. 7. Typical constant- $Q$ scans measured in $\mathrm{SrNi}_{2} \mathrm{~V}_{2} \mathrm{O}_{8}$ powder sample at $T=2 \mathrm{~K}$ in the standard three-axis mode. The solid lines are as in Fig. 5.

which can be extracted from a quantitative analysis, as described in the following section.

\section{B. Data analysis}

\section{Model cross section}

The experimental data for $\mathrm{PbNi}_{2} \mathrm{~V}_{2} \mathrm{O}_{8}$ and $\mathrm{SrNi}_{2} \mathrm{~V}_{2} \mathrm{O}_{8}$ were analyzed using a parametrized model magnetic dynamic structure factor $S(\boldsymbol{q}, \omega)$ derived within the randomphase approximation for the spin Hamiltonian 2-4, as discussed in the theory section below:

$$
\begin{aligned}
& S(\boldsymbol{Q})=P_{\|}(\boldsymbol{Q}) S_{\|}(\boldsymbol{Q})+P_{\perp}(\boldsymbol{Q}) S_{\perp}(\boldsymbol{Q}), \\
2 S_{\|}(\boldsymbol{Q})= & 2 \cos ^{2} \psi_{1} \cos ^{2} \psi_{2} S_{\|}^{\prime}(h, k, l)+\cos ^{2} \psi_{1} \sin ^{2} \psi_{2} \\
& \times\left[S_{\|}^{\prime}(h+1, k, l+1)+S_{\|}^{\prime}(h+1, k, l+3)\right] \\
& +\sin ^{2} \psi_{1} \cos ^{2} \psi_{2}\left[S_{\|}^{\prime}(h, k+1, l+1)+S_{\|}^{\prime}(h, k+1, l\right. \\
& +3)]+2 \sin ^{2} \psi_{1} \sin ^{2} \psi_{2} S^{\prime}(h+1, k+1, l+2),
\end{aligned}
$$

$$
\begin{aligned}
2 S_{\perp}(\boldsymbol{Q})= & 2 \cos ^{2} \psi_{1} \cos ^{2} \psi_{2} S_{\perp}^{\prime}(h, k, l)+\cos ^{2} \psi_{1} \sin ^{2} \psi_{2} \\
& \times\left[S_{\perp}^{\prime}(h+1, k, l+1)+S_{\perp}^{\prime}(h+1, k, l+3)\right] \\
& +\sin ^{2} \psi_{1} \cos ^{2} \psi_{2}\left[S_{\perp}^{\prime}(h, k+1, l+1)+S_{\perp}^{\prime}(h, k+1, l\right. \\
& +3)]+2 \sin ^{2} \psi_{1} \sin ^{2} \psi_{2} S^{\prime}(h+1, k+1, l+2), \quad(7)
\end{aligned}
$$

$$
\begin{aligned}
& \psi_{1}=\frac{\pi d}{a} h, \\
& \psi_{2}=\frac{\pi d}{a} k .
\end{aligned}
$$

In these formulas the argument $\omega$ has been dropped. The phases $\psi_{1}$ and $\psi_{2}$ represent the 3D structure factor of the spiral-shaped spin chains, and $d=0.08 a$ is the offset of each $\mathrm{Ni}^{2+}$-ion relative to the central axis of the corresponding spiral chain, along the $a$ or $b$ axis. The polarization factors for longitudinal (polarization along the $c$ axis) and transverse [polarization in the $(a, b)$ plane] spin excitations are defined as

$$
\begin{gathered}
P_{\|}(\boldsymbol{Q})=\sin ^{2}(\widehat{\boldsymbol{Q}, \boldsymbol{z}}), \\
P_{\perp}(\boldsymbol{Q})=1+\cos ^{2}(\widehat{\boldsymbol{Q}, \boldsymbol{z}}) .
\end{gathered}
$$

The dynamic structure factors $S_{\|}^{\prime}$ and $S_{\perp}^{\prime}$ for straight (as opposed to spiral-shaped) Haldane chains are written in the single-mode approximation(SMA): ${ }^{12-14}$

$$
\begin{gathered}
S_{\|}^{\prime}(\boldsymbol{Q}, \omega)=\frac{1-\cos (\boldsymbol{Q c} / 4)}{2} \frac{Z v}{\hbar \omega_{\|}(\boldsymbol{Q})} \delta\left(\hbar \omega-\hbar \omega_{\|}(\boldsymbol{Q})\right), \\
S_{\perp}^{\prime}(\boldsymbol{Q}, \omega)=\frac{1-\cos (\boldsymbol{Q c} / 4)}{2} \frac{Z v}{\hbar \omega_{\perp}(\boldsymbol{Q})} \delta\left(\hbar \omega-\hbar \omega_{\perp}(\boldsymbol{Q})\right) .
\end{gathered}
$$

Here $v=2.49 \mathrm{~J}$ is the spin-wave velocity, and $Z=1.26$ [Ref. 15 , Eq. (8.2). Note that in this notation $Z$ is denoted as $g$ ]. Finally, the dispersion relation for weakly coupled chains are given by

$$
\begin{aligned}
{\left[\hbar \omega_{\|}(\boldsymbol{Q})\right]^{2}=} & \Delta_{\|}^{2}+v^{2} \sin ^{2}(\pi l / 2)-\frac{1}{2} Z v J_{1, \|} \cos (\pi l / 2) \\
& \times[\cos (\pi h)+\cos (\pi k)](1-\cos \pi l / 2),(1) \\
{\left[\hbar \omega_{\perp}(\boldsymbol{Q})\right]^{2}=} & \Delta_{\perp}^{2}+v^{2} \sin ^{2}(\pi l / 2)-\frac{1}{2} Z v J_{1, \perp} \cos (\pi l / 2) \\
& \times[\cos (\pi h)+\cos (\pi k)](1-\cos \pi l / 2),
\end{aligned}
$$

where $\Delta_{\|}$and $\Delta_{\perp}$ are the longitudinal and transverse intrinsic Haldane gaps for noninteracting chains, respectively. The splitting of the triplet in our model is caused by single-ion anisotropy: ${ }^{16}$

$$
\begin{gathered}
\Delta_{\perp}=\langle\Delta\rangle-0.57 D, \\
\Delta_{\|}=\langle\Delta\rangle+1.41 D .
\end{gathered}
$$

An important parameter is the mean intrinsic Haldane gap $\langle\Delta\rangle \approx\left(\Delta_{\|}+2 \Delta_{\perp}\right) / 3$. This parameter is, to a good approximation, defined by $J$ alone: $\langle\Delta\rangle \approx 0.41 J$. It is also useful to define the actual gaps (excitation energies at the 3D AF zone center):

$$
E_{\min , \perp}^{2}=\Delta_{\perp}^{2}-2 Z v\left|J_{1, \perp}\right|,
$$




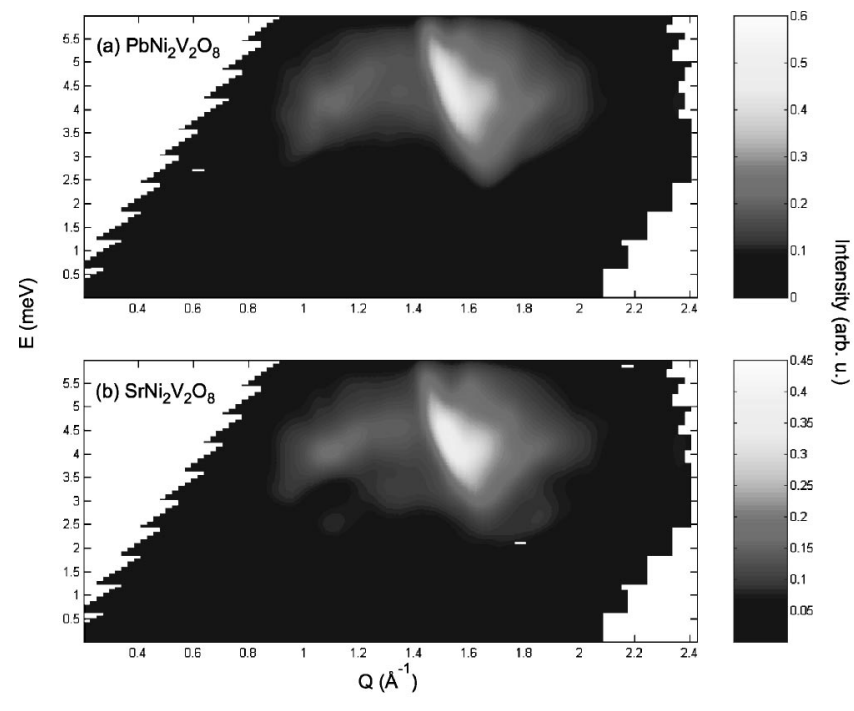

FIG. 8. Simulated inelastic scattering cross section based on parameters obtained in a global fit to the ASD data for $\mathrm{PbNi}_{2} \mathrm{~V}_{2} \mathrm{O}_{8}$ (a) and $\mathrm{SrNi}_{2} \mathrm{~V}_{2} \mathrm{O}_{8}$ (b) at $T=1.5 \mathrm{~K}$.

$$
E_{\min , \|}^{2}=\Delta_{\|}^{2}-2 Z v\left|J_{1, \|}\right|
$$

The spherical average of Eq. (5) was calculated numerically using a Monte Carlo algorithm that also eliminates the $\delta$ functions in Eqs. (12) and (13). The parameters were then refined by a standard least-squares routine to best-fit the data. To accelerate the fitting process the ASD data were resampled to a rectangular $0.046 \AA^{-1} \times 0.1 \mathrm{meV}$ resolution. An additional benefit of this resampling is that it allowed us not to worry about resolution effects. The lower $0.5 \mathrm{meV}$ energy transfer range, that contains the elastic-incoherent and possibly phonon scattering, was excluded from the fits.

\section{Analysis of $\mathrm{PbNi}_{2} \mathrm{~V}_{2} \mathrm{O}_{8}$ data}

For the $\mathrm{Pb}$ compound the adjustable parameters were the two intrinsic gap energies $\Delta_{\|}$and $\Delta_{\perp}$, and the doublet 3D gap $E_{\min , \perp}$. The singlet $3 \mathrm{D}$ gap was fixed to $E_{\text {min, }}$ $=1.2 \mathrm{meV}$, as determined in high-field bulk measurements. ${ }^{9}$ The only additional parameter was an overall scale factor. The least-squares refinement yields $\Delta_{\perp}=4.0 \pm 0.25 \mathrm{meV}$, $\Delta_{\|}=3.1 \pm 0.3 \mathrm{meV}$, and $E_{\perp}^{(\mathrm{min})}=2.4 \pm 0.2 \mathrm{meV}\left(J_{1}<0\right)$, with $\chi^{2}=2.3$. The resulting fit is shown in Fig. 8. Substituting the obtained values into the expression for $S(Q, \omega)$, performing a powder average and convoluting the result with the spectrometer resolution function, reproduces the measured threeaxis const- $E$ and const- $Q$ scans rather well, as shown in solid lines in Figs. 5(a) and 6. The relatively large $\chi^{2}$ of the global fit to the ASD data is to be attributed to systematic error, primarily due to "spurious" scattering. In particular, the data are partially contaminated by spurions of type " $k_{i} \rightarrow k_{i}$ ", and ' $k_{f} \longrightarrow k_{f}$ "' originating from the stronger Bragg powder lines from the sample. Areas that are affected by these spurious processes are shown as shaded "streaks" in Fig. 4. Another prominent streak in the data is to the left from, and parallel to, the two shaded areas. The origin of this feature is not known. It is however resolution-limited and temperature independent, and is thus almost certainly spurious and of nonmagnetic origin. Considering that this type of systematic error is unavoidable in powder experiments, the obtained model fit to the data is quite acceptable.

To illustrate that the $J_{1}$ is in fact a relevant parameter, we have performed simulations of the dynamic structure factors using the same values $\Delta_{\perp}=4.0 \mathrm{meV}, \Delta_{\|}=3.1 \mathrm{meV}$, as determined in the analysis of the ASD data, but assuming $J_{1, \perp}$ $=J_{1, \|}=0$. This results is represented in Fig. 5 by a dashed line. The well-defined intensity maximum seen in the data at $|Q| \approx 1.2 \AA^{-1}$, is replaced with a broad monotonous feature for uncoupled chains. In contrast, the interacting chain model reproduces the peak rather well.

The parameters $E_{\min , \perp}$ and $E_{\min , \|}$ are dependent only on the absolute values of $J_{1, \perp}$ and $J_{1, \|}$. However, the sign of these coupling constants explicitly enters Eqs. (14) and (15), and thus directly affects $S(\boldsymbol{q}, \boldsymbol{\omega})$. Separate fits to the data were performed assuming positive or negative signs. The values quoted in the previous paragraph correspond to $J_{1, \perp}$ $<0$ and $J_{1, \|}<0$. Assuming positive signs leads to a total incompatibility between the experimental data and model cross section. A negative sign of $J_{1}$, that links pairs of spins offset relative to each other along the chain axis, means that at the mean-field level the effective inter-chain coupling is antiferromagnetic, and suggest that the 3D magnetic zone center (the global minimum of the 3D dispersion relation) is located at the $(1,1,2)$ reciprocal-space point. Using the fit results described above, from Eqs. (18), (19) we get $J_{1, \perp}=$ $-0.18 \mathrm{meV}$ and $J_{1, \|}=-0.14 \mathrm{meV}$. Note that $\left|J_{1}\right|$ is larger by a factor of 1.5-2, compared to our previous estimate $\left(\left|J_{\perp}\right|=0.096 \pm 0.003 \mathrm{meV}\right)$ in Ref. 9. This discrepancy should be partly attributed to a difference in the definition of $J_{1}$. In our previous model each spin was coupled to four spins in adjacent chains (coordination number 4). In the present model the interchain coordination number is 2 . This automatically translates into a factor of 2 for $\left|J_{1}\right|$ within the random phase approximation.

From the refined $\Delta_{\perp}$ and $\Delta_{\|}$we can also get the in-chain coupling constant: $J \approx 9.0 \mathrm{meV}$. This value is in a better agreement with the high-temperature susceptibility estimate $J \approx 8.2 \mathrm{meV}$ in Ref. 9, than our preliminary neutron result $J$ $=9.5 \mathrm{meV}$, from the same reference. The anisotropy constant $D$ can be estimated from $\Delta_{\perp}$ and $\Delta_{\|}$using Eqs. (16) and (17). For $\mathrm{PbNi}_{2} \mathrm{~V}_{2} \mathrm{O}_{8}$ we get $D=-0.45 \mathrm{meV}$.

To determine the relevance of the two-ion anisotropy of $J_{1}$, we performed additional fits to the data, assuming $J_{1, \perp}$ $\equiv J_{1, \|}$, and varying this parameter together with the intrinsic gap energies. The best fit with this model corresponds to $\chi^{2}=2.7$ and is thus in considerably worse agreement with our measurements than the anisotropic- $J_{1}$ model. At a glance, it may appear surprising that our refinement gives $\left|J_{1, \perp}\right|>\left|J_{1, \|}\right|$ (easy-plane type), while $D<0$ (easy axis). This, however, is not necessarily a contradiction. Indeed, $D$, which is expected to have a large contribution from single-ion anisotropy, is primarily determined by spin-orbit interactions on the magnetic sites themselves. In contract, the anisotropy of $J_{1}$ is influenced by spin-orbit coupling through the entire exchange route, that in our model involves the $\mathrm{VO}_{4}$ tetrahedra. Single-ion and two-ion anisotropies can thus be considered as independent parameters.

\section{Analysis of $\mathrm{SrNi}_{2} \mathrm{~V}_{2} \mathrm{O}_{8}$, data}

Similar data analysis was performed for $\mathrm{SrNi}_{2} \mathrm{~V}_{2} \mathrm{O}_{8}$. $E_{\text {min, },}$ in this case was fixed at zero value (see discussion in 
the theory section below). The best fit to the ASD data is obtained with $\Delta_{\perp}=3.9 \pm 0.3 \mathrm{meV}, \Delta_{\|}=2.8 \pm 0.4 \mathrm{meV}$ and $E_{\text {min }, \perp}=2.35 \pm 0.3 \mathrm{meV}$ with $\chi^{2}=2.0$, and is shown in Fig. $8(\mathrm{~b})$. These values correspond to interchain coupling constants $J_{1, \perp}=-0.18 \mathrm{meV}$ and $J_{1, \|}=-0.15 \mathrm{meV}$, respectively, almost identical to the corresponding $\mathrm{PbNi}_{2} \mathrm{~V}_{2} \mathrm{O}_{8}$ values. An important consistency check is that using the gap values to estimate $J$ gives almost the same value as for $\mathrm{PbNi}_{2} \mathrm{~V}_{2} \mathrm{O}_{8}$, as expected: $J=8.6 \mathrm{meV}$. The easy-axis anisotropy constant in $\mathrm{SrNi}_{2} \mathrm{~V}_{2} \mathrm{O}_{8}$ is larger than in $\mathrm{PbNi}_{2} \mathrm{~V}_{2} \mathrm{O}_{8}: D=-0.56 \mathrm{meV}$. Simulations for constant $E$ and constant- $Q$ scans measured for $\mathrm{SrNi}_{2} \mathrm{~V}_{2} \mathrm{O}_{8}$ in the standard three-axis mode are shown in solid lines in Figs. 5(b) and 7.

\section{Scattering at the 3D magnetic zone center}

From the point of view of spin dynamics, the main distinction between the singlet-ground-state $\mathrm{PbNi}_{2} \mathrm{~V}_{2} \mathrm{O}_{8}$ and the magnetically ordered $\mathrm{SrNi}_{2} \mathrm{~V}_{2} \mathrm{O}_{8}$ is expected to be the presence of a spin gap in the former system, and a gapless excitation spectrum in the latter. In principle, the gap is directly accessible experimentally at the 3D AF zone center, where the dispersion of magnetic excitations is a global minimum. As mentioned above, for both vanadates the $3 \mathrm{D}$ zone center is at $\boldsymbol{Q}^{(0)}=(1,1,2)$, which corresponds to a momentum transfer $\left|\boldsymbol{Q}^{(0)}\right| \approx 1.67 \AA^{-1}$. Constant- $Q$ scans extracted from our ASD data for this momentum transfer, as well as a standard $E_{f}=5 \mathrm{meV}$ constant $Q$ scan at this wave vector, are shown in Fig. 9. The solid line is a simulation based on the parameter values cited above. To better understand these data, we note that near the $3 \mathrm{D}$ zone center the single-mode contribution to the dynamic structure factor, independently of the details of the spin Hamiltonian, should be of the following form:

$$
\begin{gathered}
S(\boldsymbol{Q}, \omega) \propto \frac{1}{\omega_{Q}} \delta\left(\omega-\omega_{Q}\right), \\
\left(\hbar \omega_{Q}\right)^{2}=\Delta^{2}+v_{\|}^{2}\left(Q_{\|}-Q_{\|}^{(0)}\right)^{2}+v_{\perp}^{2}\left(Q_{\perp}-Q_{\perp}^{(0)}\right)^{2} .
\end{gathered}
$$

In this formula $\Delta$ is the gap energy, and $v_{\|}$and $v_{\perp}$ are spinwave velocities along and perpendicular to the chain axis $c$, respectively. The data shown in Fig. 9(c) were collected with a horizontally focusing analyzer. These intensities were normalized by incoherent scattering from the two systems, and thus can directly compared. At energy transfers below $1 \mathrm{meV}$ in this mode we are picking up a great deal of diffuse and phonon scattering $[(1,1,2)$ is an allowed nuclear Bragg peak]. Above this contaminated region though, in a powder sample we effectively observe a $\boldsymbol{Q}$ integral of the cross section around the $3 \mathrm{D}$ zone center. The same applies to the scans in Figs. 9(a) and 9(b), where integration was performed in the range $1.6-1.7 \AA^{-1}$. For the $Q$-integrated intensity Eq. (20) gives

$$
S(\omega) \propto \sqrt{\omega^{2}-\Delta^{2}} .
$$

The linear increase of intensity seen for $\mathrm{SrNi}_{2} \mathrm{~V}_{2} \mathrm{O}_{8}$ in Fig. 9 is thus consistent with having a gapless spin wave, but of course the presence of a gap of less than $1 \mathrm{meV}$ cannot be excluded. In contrast, for $\mathrm{PbNi}_{2} \mathrm{~V}_{2} \mathrm{O}_{8}$, the threshold behavior, and a smaller absolute intensity between 1 and $2 \mathrm{meV}$
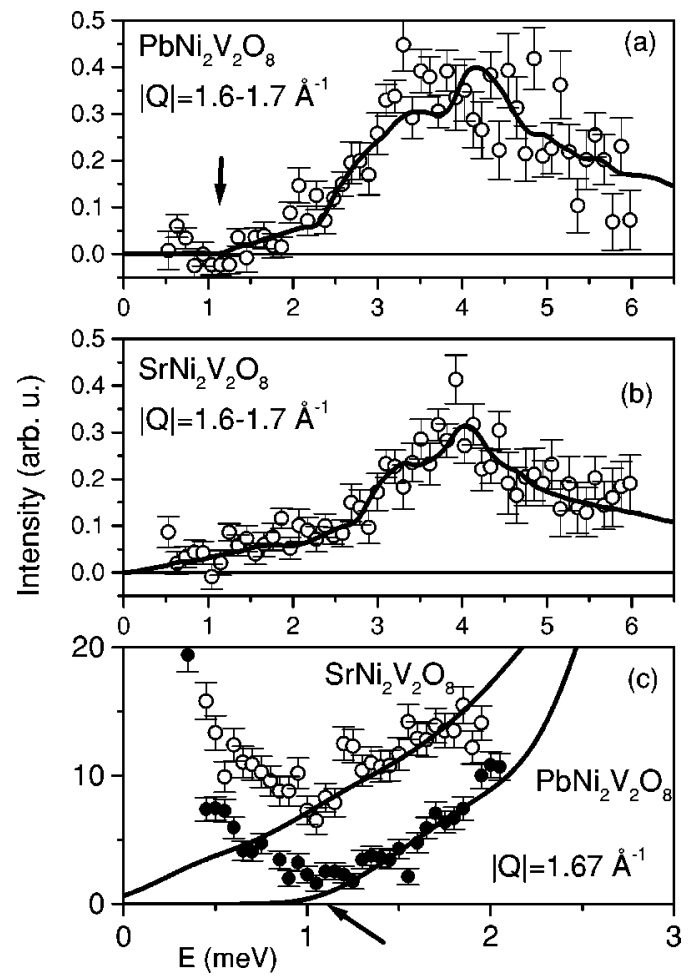

FIG. 9. Constant- $Q$ scans extracted from the ASD data sets for $\mathrm{PbNi}_{2} \mathrm{~V}_{2} \mathrm{O}_{8}$ (a) and $\mathrm{SrNi}_{2} \mathrm{~V}_{2} \mathrm{O}_{8}$ (b) at a momentum transfer that corresponds to the $3 \mathrm{D}$ antiferromagnetic zone-center at $T=1.5 \mathrm{~K}$ (symbols). Unlike in $\mathrm{SrNi}_{2} \mathrm{~V}_{2} \mathrm{O}_{8}$, the measured inelastic intensity in $\mathrm{PbNi}_{2} \mathrm{~V}_{2} \mathrm{O}_{8}$ extrapolates to zero at a nonzero energy transfer (arrows). This is even better seen in constant- $Q$ scans measured in the standard three-axis mode at $T=2 \mathrm{~K}$ (c). Open and solid symbols correspond to $\mathrm{SrNi}_{2} \mathrm{~V}_{2} \mathrm{O}_{8}$ and $\mathrm{PbNi}_{2} \mathrm{~V}_{2} \mathrm{O}_{8}$, respectively. In the latter plot the data for the two compounds were brought to a single scale using the elastic incoherent signal. In all cases the solid lines are as in Fig. 5.

energy transfer, are in agreement with our expectations for a gap spectrum. These effects are also seen in constant- $Q$ scans extracted from the ASD data by resampling the pixels in a $0.1 \AA^{-1} Q$-range. Clearly, further measurements on single crystal or, at least, aligned-powder samples, are required to unambiguously resolve the gap/no gap issue for $\mathrm{PbNi}_{2} \mathrm{~V}_{2} \mathrm{O}_{8}$ and $\mathrm{SrNi}_{2} \mathrm{~V}_{2} \mathrm{O}_{8}$.

\section{C. $\mathrm{SrNi}_{2} \mathrm{~V}_{2} \mathrm{O}_{8}$ : Temperature dependence}

To better understand the mechanism of long-range ordering of $\mathrm{SrNi}_{2} \mathrm{~V}_{2} \mathrm{O}_{8}$ we studied the temperature dependence of inelastic scattering at the momentum transfer $\left|Q^{(0)}\right|$ $=1.67 \AA^{-1}$ in this compound. Typical data are shown in Fig. 10. The solid lines were obtained by fitting our model cross section to the data at each temperature. The lower $0.75 \mathrm{meV}$ energy transfer range was excluded from this analysis, as the difficult-to-estimate phonon contribution in this range is expected to increase dramatically with increasing $T$. The set of independent parameters was slightly modified. The anisotropy splitting of the triplet $D$, as well as both interchain coupling constants $J_{1, \perp}$ and $J_{1, \|}$ were fixed at the values determined at $T=1.5 \mathrm{~K}$. The mean gap $\langle\Delta\rangle$ and an intensity prefactor were refined to best-fit the scans at each temperature. The obtained temperature dependences are shown in 

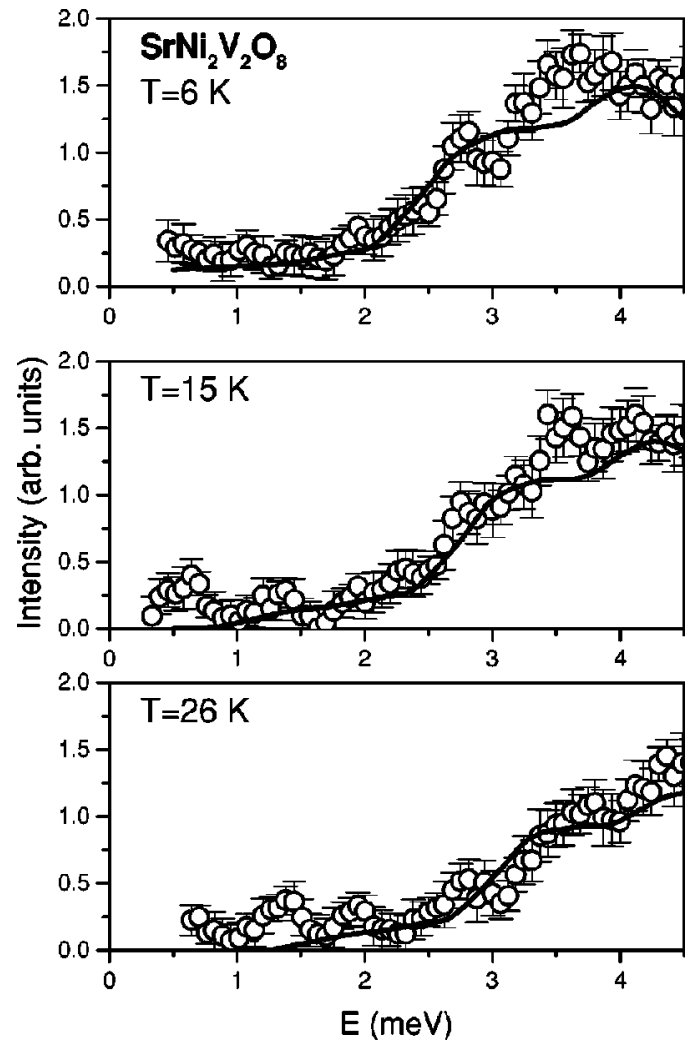

FIG. 10. Typical constant- $Q$ scans measured in $\mathrm{SrNi}_{2} \mathrm{~V}_{2} \mathrm{O}_{8}$ powder at different temperatures, at a momentum transfer that corresponds to the $3 \mathrm{D}$ antiferromagnetic zone-center. The solid lines are fits to the model cross section, as described in the text.

Fig. 11. As previously observed in other Haldane-gap systems, $\langle\Delta\rangle$ increases with increasing $T$. Even though this change is rather small, according to Eqs. (18) and (19), it corresponds to an appreciable variation of the gap in the longitudinal mode (Fig. 12).

\section{THEORY AND DISCUSSION}

\section{A. Derivation of the model cross section}

To calculate the effect of weak interchain interactions on dynamic spin correlations in $\mathrm{PbNi}_{2} \mathrm{~V}_{2} \mathrm{O}_{8}$ and $\mathrm{SrNi}_{2} \mathrm{~V}_{2} \mathrm{O}_{8}$ we shall use the random phase approximation (RPA). ${ }^{17}$ This approach for directly coupled Haldane spin chains has been successfully applied, for example, to $\mathrm{CsNiCl}_{3}$ (Ref. 4). More recently it was shown to also work well for Haldane chains coupled via classical spins. ${ }^{18}$ The only additional difficulties in the present case arise from the complicated 3D arrangement of magnetic ions in the $\mathrm{PbNi}_{2} \mathrm{~V}_{2} \mathrm{O}_{8}$ structure, and from the rather nontrivial geometry of interchain coupling. To somewhat simplify the task we chose to break it up into two distinct problems. First, we concerned ourselves only with the topology of magnetic interactions and consider an equivalent Bravais lattice of spins, assuming straight spin chains and a system of inter-chain bonds shown in Fig. 3(b). In a separate step we adapted the result to the more complex structure of $\mathrm{PbNi}_{2} \mathrm{~V}_{2} \mathrm{O}_{8}$ and $\mathrm{SrNi}_{2} \mathrm{~V}_{2} \mathrm{O}_{8}$.

The bare (noninteracting) susceptibility for an isolated Haldane spin chain is known to be rather well described by the single-mode approximation (SMA): ${ }^{12-14}$
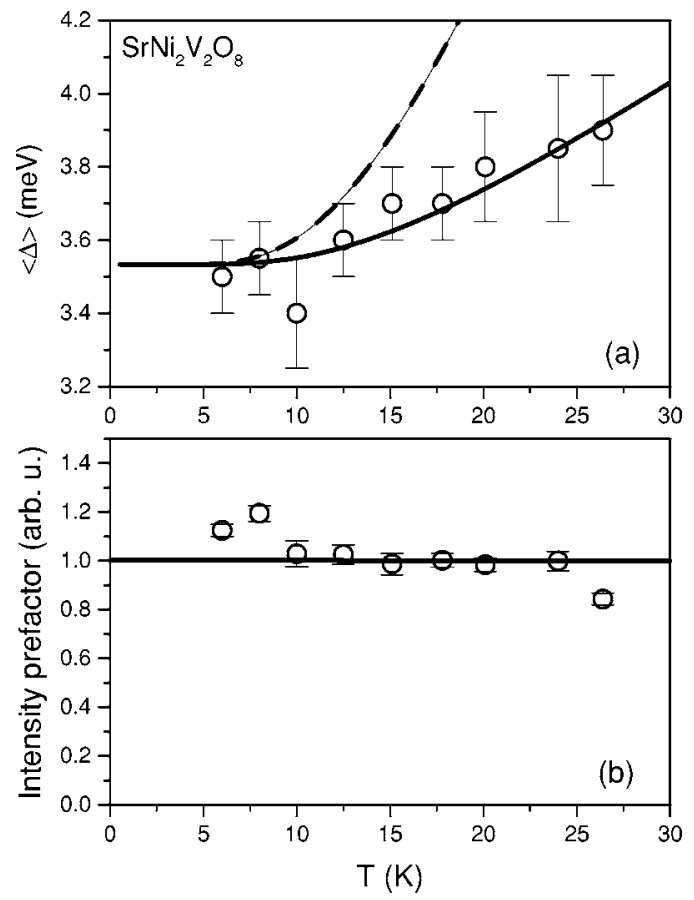

FIG. 11. Measured temperature dependence of the mean intrinsic Haldane gap $\langle\Delta\rangle$ (a) and intensity prefactor in the model cross section described in the text (b) for $\mathrm{SrNi}_{2} \mathrm{~V}_{2} \mathrm{O}_{8}$.

$$
\begin{aligned}
\chi_{0}(\boldsymbol{Q}, \omega)= & \frac{1-\cos \left(Q_{z} c / 4\right)}{2} \\
& \times \frac{Z v}{\Delta^{2}+v^{2} \sin ^{2}\left(Q_{z} c / 4\right)-(\hbar \omega+i \epsilon)^{2}} .
\end{aligned}
$$

This expression should be used separately for each channel of spin polarization, with appropriate values of gap energy $\Delta$ for each particular mode. Writing down the exact RPA equations for the model shown in Fig. 3(b) is straightforward, but rather tedious, due to the fact that interchain bonds connect sites with different chain-axis cell coordinates. For the particular crystal structure, for each momentum transfer $\boldsymbol{q}$, this leads to RPA coupling between $\chi(\boldsymbol{q})$ and $\chi\left(\boldsymbol{q}+n \boldsymbol{c}^{*}\right)(n$ $=1,2,3)$. The resulting RPA susceptibility contains a multitude of spin-wave branches. The calculation can be made much simpler for low-energy excitations, that involve only

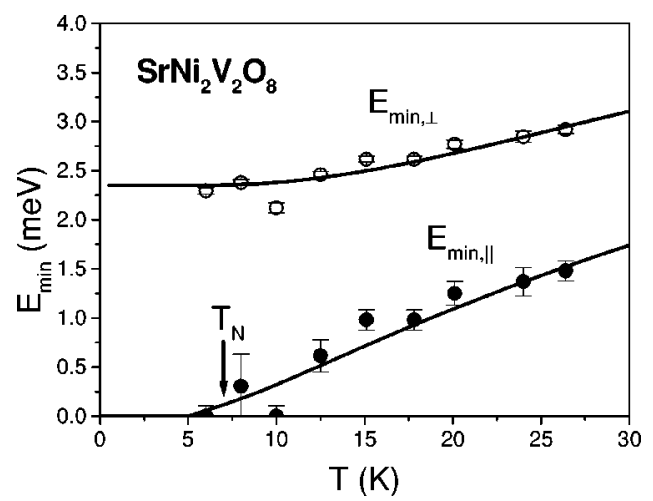

FIG. 12. Measured temperature dependence of the 3D gap energies in for $\mathrm{SrNi}_{2} \mathrm{~V}_{2} \mathrm{O}_{8}$. The solid lines are as in Fig. 11 . 
momentum transfers close to the $1 \mathrm{D}$ AF zone center. At these wave vectors the spectrum is dominated by a single branch for each channel of spin polarization, and the RPA dynamic sysceptibility given by

$$
\chi_{\mathrm{RPA}}^{-1}(\boldsymbol{Q})=\chi_{0}^{-1}(\boldsymbol{Q})\left[\chi_{0}(\boldsymbol{Q}) \mathcal{J}(\boldsymbol{Q})+1\right],
$$

where

$$
\mathcal{J}(\boldsymbol{Q})=J_{1} \cos \left(Q_{z} c / 4\right)\left[\cos \left(Q_{x} a / 2\right)+\cos \left(Q_{y} a / 2\right)\right]
$$

is the combined Fourier transform of all interchain interactions involving magnetic sites in a crystallographic unit cell. The above RPA equation is easily solved analytically. By taking the imaginary part of the thus obtained $\chi_{\mathrm{RPA}}(\boldsymbol{Q})$ we arrive at Eqs. (12)-(15).

Note that at this level approximation [Eq. (23)] the actual geometry of interchain interactions becomes unimportant. For example, for the geometry of Fig. 3(a), the result would be the same except that the prefactor $\cos \left(Q_{z} c / 4\right)$ in Eq. (24) is replaced by a constant factor of 2 (all interchain bonds are perpendicular to the chains and the interchain coordination number is twice as large). As low-energy excitations are all located around $Q_{z} c / 4 \approx \pi$, the two models are indistinguishable in our experiments.

The transformation from the Bravais spin lattice (straight chains) to the spiral-chain structure of $\mathrm{PbNi}_{2} \mathrm{~V}_{2} \mathrm{O}_{8}$ and $\mathrm{SrNi}_{2} \mathrm{~V}_{2} \mathrm{O}_{8}$, while tedious, is quite straightforward as well. The final results is given by Eqs. (6) and (9). In each spiralshaped chain the transverse displacement of magnetic sites from the chain-axis has a step-4 periodicity. As a result, at any wave vector $(h, k, l)$ one simultaneously observes straight-chain scattering calculated at wave vectors $(h, k, l$,$) ,$ $(h+1, k, l \pm 1),(h, k+1, l \pm 1)$ and $(h+1, k+1, l+2)$, each with a particular structure factor, depending on the momentum transfer perpendicular to the chain axis. In addition to $(1,1,2)$, wave vectors $(2,2,0),(1,0,1)$ and $(0,1,1)$ will also correspond to global minima of 3D spin-wave dispersion, and magnetic Bragg reflections can be expected to occur at these reciprocal-space points in the ordered phase of $\mathrm{SrNi}_{2} \mathrm{~V}_{2} \mathrm{O}_{8}$.

\section{B. Temperature effects}

\section{Disordered phase}

The RPA calculation above can be repeated almost verbatim for the case $T>0$. Temperature enters the RPA equations indirectly, through an intrinsic temperature dependence of bare susceptibilities of individual chains [Eq. (22)]. Two effects at $T>0$ need be considered: (i) the increase of the Haldane gap, compared to its $T=0$ value, and (ii) damping of Haldane excitations. Both phenomena have been observed in a number of model quasi-1D systems (see, for example, Refs. 19-21). Unfortunately, it is almost impossible to extract meaningful information regarding excitation lifetimes from powder inelastic data. As most of the observed inelastic signal originates from excitations with energies greater than $2 \mathrm{meV}$, the temperature dependence of the intrinsic Haldane gap is much easier to observe. For this reason we analyzed the temperature dependence of the inelastic signal measured in $\mathrm{SrNi}_{2} \mathrm{~V}_{2} \mathrm{O}_{8}$ using the same single-mode approximation [Eq. (22)], as at base temperature (see fits in Fig. 10), to obtain the $T$ dependences of intrinsic Haldane gap energies (Fig. 11). A self-consistency check is that the refined intensity prefactor is almost $T$ independent, as shown in Fig. 11(b). In other words, the decrease of actual excitation intensity is entirely due to the increase of gap energy, and the intensity scales as $1 / \omega$.

From the mapping of the Heisenberg Hamiltonian on the quantum nonlinear $\sigma$ model (NLSM) one expects a rather steep increase of the gap energy with increasing temperature: $^{22}$

$$
\Delta(T) \approx \Delta(0)+\sqrt{2 \pi} \sqrt{T \Delta(0)} \exp \left(-\frac{\Delta(0)}{k_{\mathrm{B}} T}\right) .
$$

The actual increase of the gap energy observed experimentally, NENP $\left(\mathrm{Ni}\left(\mathrm{C}_{2} \mathrm{H}_{8} \mathrm{~N}_{2}\right)_{2} \mathrm{NO}_{2} \mathrm{ClO}_{4}\right),{ }^{19}$ NINAZ $\left(\mathrm{Ni}\left(\mathrm{C}_{3} \mathrm{H}_{10} \mathrm{~N}_{2}\right)_{2} \mathrm{~N}_{3}\left(\mathrm{ClO}_{4}\right)\right),{ }^{20}$ and $\mathrm{Y}_{2} \mathrm{BaNiO}_{5},{ }^{21}$ was found to be consistently smaller than this NLSM prediction. The suppressed $T$ dependence of the gap energy is also observed in $\mathrm{SrNi}_{2} \mathrm{~V}_{2} \mathrm{O}_{8}$. For comparison with our data, Eq. (25) is plotted in a dashed line in Fig. 11(a).

The temperature dependence of $\Delta_{\perp}$ and $\Delta_{\|}$, deduced from the fits to the data at each temperature, and shown in Fig. 12, suggests a softening of the $c$-axis polarized gap mode between 5 and $10 \mathrm{~K}$, i.e., approximately at $T_{N}=7 \mathrm{~K}$. This result is very reassuring and suggests we are indeed dealing with the same type of soft-mode mechanism of magnetic ordering in coupled gapped spin chains, as seen in the well-studied $\mathrm{CsNiCl}_{3}$ compound. ${ }^{3-5}$ The fact that it is the $c$-axis mode that softens is consistent with the dominance of easy-axis anisotropy in the system.

\section{Placement on the phase diagram and magnetically ordered state in $\mathrm{SrNi}_{2} \mathrm{~V}_{2} \mathrm{O}_{8}$}

The line of quantum phase transition separating the ordered and spin-liquid states can be derived from Eqs. (16)(19). The critical value for $J_{1}$ corresponds to the lower gap energy (in our case $E_{\text {min, }}$ ) being equal to zero. The resulting phase diagram is very similar to the direct numerical calculations by Sakai and Takashi, ${ }^{7}$ and is shown in Fig. 1. It is clear that in our case one cannot unambiguously extract any detailed information on interchain interactions from powder experiments. What is relevant for the phase behavior though is the effective MF interchain coupling constant, i.e., the product of $J_{1}$ and the interchain coordination number. The corresponding values obtained in our analysis of the powder data are in very good agreement with those obtained in previous bulk studies, and thus inspire enough confidence to attempt placing $\mathrm{PbNi}_{2} \mathrm{~V}_{2} \mathrm{O}_{8}$ on the phase diagram. Using experimental values for $J, D$, and $J_{1}$, we can tentatively position this compound on Fig. 1 (solid circle), just below the spin liquid- Ising transition line.

Placing the $\mathrm{Sr}$ compound on the phase diagram is more difficult. Our model cross section was derived under the implicit assumption that the system is in a nonmagnetic state, which is not applicable to $\mathrm{SrNi}_{2} \mathrm{~V}_{2} \mathrm{O}_{8}$ at $T<T_{\mathrm{N}}$. In this regime one expects an increase of the gap energy for the longitudinal mode, due to the presence of a mean static staggered field generated by the ordered staggered moment in the system. $^{23,24}$ To adapt the MF-RPA calculation to this temperature regime one has to know the ordered moment in the 
system. To date, powder diffraction experiments on $\mathrm{SrNi}_{2} \mathrm{~V}_{2} \mathrm{O}_{8}$ failed to detect any magnetic Bragg reflections in this compound at low temperatures, ${ }^{25}$ and the magnetic order parameter is thus expected to be very small. Provided the ordered moment is small, within the accuracy of the powder experiment, our analysis based on the assumption $E_{\min , \|}=0$ is justified to get the overall picture of interchain interactions. However, the extracted values for $J_{1}$ and the intrinsic gap energies cannot be considered sufficiently accurate to place the material on the phase diagram. Indeed, the assumption $E_{\text {min, }}=0$ implies that the system is right on the phase transition line and $T_{\mathrm{N}} \equiv 0$. In fact, $\mathrm{SrNi}_{2} \mathrm{~V}_{2} \mathrm{O}_{8}$ must be deeper in the ordered region of the phase diagram, than suggested by the values for $J_{1}$ and $\Delta$ deduced from fits to the $T=1.5 \mathrm{~K}$ neutron data. This fact is indicated by an arrow in Fig. 1. Note that the analysis for $T>T_{N}$, where the excitations are expected to have a nonzero energy gap, is not affected by the above-mentioned artifact.

\section{SUMMARY}

The main result of this work is that a simple model with a minimal number of adjustable parameters is consistent with both the previously published bulk data and new inelastic neutron-scattering results. This brings strong support to the idea that $\mathrm{PbNi}_{2} \mathrm{~V}_{2} \mathrm{O}_{8}$ and $\mathrm{SrNi}_{2} \mathrm{~V}_{2} \mathrm{O}_{8}$ should be considered as Haldane-gap antiferromagnets in which interchain interactions and magnetic anisotropy are just strong enough to bring them close to the line of quantum phase transition between the spin-liquid and Ising-like states. For the Pb-based compound, the knowledge that the ground state is indeed disordered, makes the data analysis more straightforward, and the determined parameters can be used to position this com- pound on the Sakai-Takahashi phase diagram. For the magnetically ordered $\mathrm{SrNi}_{2} \mathrm{~V}_{2} \mathrm{O}_{8}$ the observed $T$ dependence of inelastic scattering indicates that long-range order is a result of a soft-mode transition, much like in $\mathrm{CsNiCl}_{3}$. A totally new piece of information obtained in the present study is the sign of transverse dispersion of magnetic excitations. The effective MF interchain coupling is antiferromagnetic, and the $3 \mathrm{D}$ magnetic zone center is located at the $(1,1,2)$ reciprocal-space point. It is at this position that magnetic Bragg scattering should occur in the ordered $\mathrm{SrNi}_{2} \mathrm{~V}_{2} \mathrm{O}_{8}$ compound.

For a complete characterization of these new and exciting quasi-1D systems, future aligned-powder and/or single-crystal experiments will be required. High-pressure studies, as previously attempted for NENP, ${ }^{26}$ may lead to an observation of pressure-induced long-range ordering in a quantumdisordered magnet.

\section{ACKNOWLEDGMENTS}

We would like to thank Y. Sasago, who had suggested at the early stage of this study that $\mathrm{SrNi}_{2} \mathrm{~V}_{2} \mathrm{O}_{8}$ may be a Haldane-gap antiferromagnet. We also thank R. Wickmann for sending a copy of Ref. 11. Work at the University of Tokyo was supported in part by Grant-in-Aid for COE Research "Phase Control of Spin-Charge-Photon Coupled Systems" from the Ministry of Education, Science, Sports and Culture of Japan. Work at Brookhaven National Laboratory was carried out under Contract No. DE-AC02-76CH00016, Division of Material Science, U.S. Department of Energy. Studies at NIST were partially supported by the NSF under Contract No. DMR-9413101.
*Also at The Department of Advanced Materials Science, The University of Tokyo, Tokyo, Japan.

${ }^{\dagger}$ Present address: Central Research Institute of Electric Power Industry, 2-11-1, Iwato kita, Komae-shi, Tokyo 201-8511, Japan.

${ }^{\ddagger}$ Present address: ULSI Device Development Laboratory, NEC Corporation.

${ }^{1}$ F.D.M. Haldane, Phys. Rev. Lett. 50, 1153 (1983).

${ }^{2}$ F.D.M. Haldane, Phys. Lett. 93A, 464 (1983).

${ }^{3}$ W.J.L. Buyers et al., Phys. Rev. Lett. 56, 371 (1986); M. Enderle, Z. Tun, W.J.L. Buyers, and M. Steiner, Phys. Rev. B 59, 4235 (1999), and references therein.

${ }^{4}$ R.M. Morra, W.J.L. Buyers, R.L. Armstrong, and K. Hirakawa, Phys. Rev. B 38, 543 (1988).

${ }^{5}$ T. Inami, K. Kakurai, H. Tanaka, M. Enderle, and M. Steiner, J. Phys. Soc. Jpn. 63, 1530 (1994); M. Enderle, K. Kakurai, M. Steiner, and H. Weinfurter, J. Magn. Magn. Mater. 104-107, 809 (1992); M. Enderle et al., Europhys. Lett. 25, 717 (1994).

${ }^{6}$ I. Zaliznyak, L.-P. Regnault, and D. Petitgrand, Phys. Rev. B 50, 15824 (1994).

${ }^{7}$ T. Sakai and M. Takahashi, Phys. Rev. B 42, 4537 (1990).

${ }^{8}$ Z. Tun, W.J.L. Buyers, A. Harrison, and J.A. Rayne, Phys. Rev. B 43, 13331 (1991).

${ }^{9}$ Y. Uchiyama et al., Phys. Rev. Lett. 83, 632 (1999).

${ }^{10}$ Y. Uchiyama (unpublished).

${ }^{11}$ R. Wickman and H. Müller-Buschbaum, Rev. Chim. Minér. 23, 1 (1986).
${ }^{12}$ D.P. Arovas, A. Auerbach, and F.D.M. Haldane, Phys. Rev. Lett. 60, 531 (1988).

${ }^{13}$ G. Muller, H. Thomas, M.W. Puga, and H. Beck, J. Phys. C 14, 3399 (1981).

${ }^{14}$ S. Ma et al., Phys. Rev. Lett. 69, 3571 (1992).

${ }^{15}$ E.S. Sorensen and I. Affleck, Phys. Rev. B 49, 15771 (1994).

${ }^{16}$ O. Golinelli, T. Jolicoeur, and R. Lacaze, Phys. Rev. B 45, 9798 (1992).

${ }^{17}$ D.J. Scalapino, Y. Imry, and P. Pincus, Phys. Rev. B 11, 2042 (1975).

${ }^{18}$ A. Zheludev, S. Maslov, T. Yokoo, J. Akimitsu, S. Raymond, K. Hirota, and S.E. Nagler, Phys. Rev. B 61, 11601 (2000).

${ }^{19}$ S. Ma et al., Phys. Rev. B 51, 3289 (1995).

${ }^{20}$ A. Zheludev et al., Phys. Rev. B 53, 15004 (1996).

${ }^{21}$ T. Sakaguchi, K. Kakurai, T. Yokoo, and J. Akimitsu, J. Phys. Soc. Jpn. 65, 3025 (1996).

${ }^{22}$ T. Jolicoeur and O. Golinelli, Phys. Rev. B 50, 9265 (1994).

${ }^{23}$ I. Affleck, Phys. Rev. Lett. 62, 474 (1989).

${ }^{24}$ S. Maslov and A. Zheludev, Phys. Rev. Lett. 80, 5786 (1998).

${ }^{25} \mathrm{P}$. Boni et al. (unpublished).

${ }^{26}$ I. Zaliznyak, D.C. Dender, C. Broholm, and D.H. Reich, Phys. Rev. B 57, 5200 (1998).

${ }^{27}$ G. Xu et al., Phys. Rev. B 54, R6827 (1996).

${ }^{28}$ L.P. Regnault, I. Zaliznyak, J.P. Renard, and C. Vettier, Phys. Rev. B 50, 9174 (1994).

${ }^{29}$ H. Mutka et al., Phys. Rev. Lett. 67, 497 (1991). 